\title{
About Thought Experiment
}

\author{
Abolfazl Soltani * \\ Department of Physics, University of Birjand, Birjand, Iran \\ E-mail:soltani.a.physics@gmail.com
}

\begin{abstract}
In physics, whenever we cannot do an experiment for physical, technological, financial or ethical reasons, we use "thought experiment". In this letter we will prove that a thought experiment leads us only to possible results of an experiment not decisive result. To achieve a definitive results, an experiment must be done in the real world.
\end{abstract}

There are several thought experiments in physics; Schrodinger's cat experiment [1], EPR paradox [2] and Einstein's light box [3][4][5] are some of the most famous of them. It is clear, all experiments are initially a thought experiment. Some of them remain a thought experiment due to the human inability to perform them in the real world (because of physical, technological, financial or ethical reasons), and some of them become real physical experiments. But there is a very important point about thought experiments that seems to have been ignored so far. A thought experiment leads us only to possible results of an experiment not decisive result. To achieve a decisive results, an experiment must be done in the real world. We prove this with an example. Here we use "counterexample" method (which is mathematic method) and with a counterexample we disproof of the generalization "All thought experiments lead to the right result". A well-known example is the famous experiment of Michelson and Morley about the velocity of light [6][7][8][9]. If you do the Michelson and Morley experiment in your mind (As said every experiment initially is a thought experiment), you will conclude that the velocity of light relative to observer, depends on the velocity of observer (velocity addition law [10]). This was something that Michelson and Morley also expected [6][7]. But when their experiments were performed in the real world, they concluded that the velocity of light relative to observer, is independent of the velocity of observer. This example shows that the intellectual and empirical results of an experiment may not be the same and therefore we cannot trust to the results of a thought experiment and we cannot use its results until it is not performed in the real world. In fact, in Michelson's experiment, two events were predictable to occur: 1- shift of interference fringes (dependence of light velocity relative to observer, to the observer velocity) [8][9] and 2- non shift of interference fringes (independence of light velocity from the observer velocity) [8][9]. Only doing experiments in the real world determined which of these two results is correct. In fact, a thought experiment can only show possible results, not definitive result. Michelson and Morley's experiment, by counterexample method, proves that a thought experiment in physics is unreliable method because it give you only possible results of an experiment not decisive result. For example consider the thought experiment of rocket in General Relativity [11]. Based on this experiment, two person with the same mass, one inside a room on the ground and the other inside a rocket moving with acceleration $g$ in far space (Far from the effects of gravity of other objects), will measure the same weight if they go on the Weighing scale. But this conclusion is incorrect. The result of Michelson's experiment affects this conclusion. The result of Michelson's experiment tells us that this conclusion is not sure and we cannot comment on the decisive result until the rocket experiment is done. That is, it is possible we do rocket experiment in the real world and see that the weight of the person in the rocket is not equal to the weight of the experimenter on the weighing scale on the Earth. Let us not forget that Michelson's experiment showed that the law 
of velocity addition [11], which is quite clear and tested in our daily lives (Like sum of the velocity of two cars), is invalid in the case of light. Therefore, the inequality of the weights of two person with the same mass in rocket experiment is not stranger than the result of Michelson's experiment. Therefore it is quite expected that, in the real experiment, the person in the rocket not measure the same weight as the ground person.

50 Finally, the conclusion of this letter is that an experiment must be performed in the real world 51 so that we can use its results.

\section{References:}

53 [1]. Schrodinger, E. Die gegenwärtige Situation in der Quantenmechanik. Naturwissenschaften. volume 23, 823$54828(1935)$

55 [2]. Einstein, A. Podolsky, B. Rosen, N. Can Quantum-Mechanical Description of Physical Reality Be Considered 56 Complete? Phys. Rev. 47, 777 (1935)

57 [3]. Mcevoy, J. Oscar, Z. Introducing Quantum Theory. (Icon Books Ltd, ed. 4, 2003) pp 146-149

58 [4]. Walter, I. Einstein: His Life and Universe. (Simon \& Schuster, 2008) pp 346-347

59 [5]. Abraham, P. Subtle is the Lord: The Science and Life of Albert Einstein. (Oxford University Press, 2005) pp $60 \quad 446-448$

61 [6]. Michelson, A. Morley, E. On the Relative Motion of the Earth and the Luminiferous Ether. American Journal 62 of Science. 34 (203): 333-345 (1887)

63 [7]. Weidner, R. Sells, R. Elementary Modern Physics (Allyn and Bacon, ed. 2, 1973), pp 31-37

64 [8]. Born, M. Einstein's Theory of Relativity (Dover Publications Inc, 1962), pp 214-218

65 [9]. Resnick, R. Introduction to Special Relativity (John Wiley and Sons, Inc, 1968), pp 18-26

66 [10]. Einstein, A. Relativity: the special and the general theory (Pi Press, 2005), page 23

67 [11]. Rindler, W. Essential Relativity Special, General, and Cosmological (Springer-Verlag, ed. 2 1977), page 18 